\title{
KAP Surveys and Oral Health: A Detailed Review
}

\author{
${ }^{1}$ Ramen Haloi, ${ }^{2}$ Navin Anand Ingle, ${ }^{3}$ Navpreet Kaur
}

\begin{abstract}
'KAP' study measures the knowledge, attitude and practices of a community. It serves as an educational diagnosis of the community. The main purpose of this knowledge, attitude and practices (KAP) study is to explore changes in knowledge, attitude and practices of the community. A KAP survey is a representative study of a specific population to collect information on what is known, believed and done in relation to a particular topic. In most KAP surveys, data are collected orally by an interviewer using a structured, standardized questionnaire. These data then can be analyzed quantitatively or qualitatively depending on the objectives and design of the study. Before beginning the process of creating awareness in any given community, it is first necessary to assess the environment in which awareness creation will take place. KAP study tells us what people know about certain things, how they feel and also how they behave.
\end{abstract}

Keywords: Knowledge, Attitude, Practice and survey.

How to cite this article: Haloi R, Ingle NA, Kaur N. KAP Surveys and Oral Health: A Detailed Review. J Contemp Dent 2014;4(2):99-105.

Source of support: Nil

Conflict of interest: None

\section{INTRODUCTION}

The knowledge, attitude and practices (KAP) survey is a representative survey conducted on a particular population to identify the knowledge (K), attitudes (A) and practices (P) of a population on a specific topic - oral health in our case. In the majority of KAP studies, data are gathered orally by an interviewer who uses a structured, standardized questionnaire. These data can then be quantitatively or qualitatively analyzed according to the objectives and the ins and outs of the survey. A KAP survey can be specially designed to collect information on the issue of, but it is also possible to include general questions on practices and beliefs. ${ }^{1}$ The knowledge, attitudes and practices survey on populations for education on oral health, are also called a 'oral health KAP survey' and is a key step in the process of dissemination, adaptation and evaluation of prevention messages and programs. ${ }^{2}$

KAP surveys are highly focused evaluations that measure changes in human knowledge, attitudes and practices

\footnotetext{
${ }^{1}$ Doctor, ${ }^{2}$ Professor and Head, ${ }^{3}$ Reader

${ }^{1-3}$ Department of Public Health Dentistry, KD Dental College and Hospital, Mathura, Uttar Pradesh, India
}

Corresponding Author: Ramen Haloi, Doctor, Department of Public Health Dentistry, KD Dental College and Hospital Mathura, Uttar Pradesh, India, Phone: 09634907667, e-mail: drramenhaloi@gmail.com in response to a specific intervention, usually outreach, demonstration or education. KAP surveys have been widely used and valued around the world for at least 40 years in public health, water supply and sanitation, family planning, education and other programs. National governments, nongovernmental groups, United Nations agencies and the World Bank use KAP evaluation methods. KAP surveys are more cost-effective and resource conserving than other social research methods, because they are highly focused and limited in scope. KAP surveys tell us what people know about certain things, how they feel, and how they behave. Each KAP survey is unique to a particular setting and designed for a specific issue. Whereas social surveys may cover a wide range of social values and activities, KAP surveys focus specifically on the knowledge, attitudes and practices (behaviors) for a certain topic.

- The knowledge possessed by a community refers to their understanding of that topic.

- Attitude refers to their feelings toward this subject, as well as any preconceived ideas they may have toward it.

- Practice refers to the ways in which they demonstrate their knowledge and attitudes through their actions.

Understanding these three-dimensions will allow a project to track changes in them over time, and may enable the project to tailor activities to the needs of that community. KAP surveys should be conducted twice, both pre- and postintervention, in order to measure impact. ${ }^{3,4}$

Researchers hypothesize five stages in the adoption process: knowledge, persuasion, decision, trial, and adoption. ${ }^{5,6}$ Although, at first glance, the diffusion of innovation's stages of adoption may seem similar to Prochaska's stages of change, the stages of adoption signify a person's progress toward adopting a healthy behavior, while the stages of change indicate a person's progress toward quitting a harmful one. ${ }^{7}$ Also, the diffusion stages emphasize the role of information and sources of influence, while the stages of change emphasize cognitive dispositions. ${ }^{8}$ People become aware of new behaviors at different times. Because, they pass through the stages of adoption at varying rates, there is considerable time between the earliest and latest adopters. The spread of new ideas and practices can be graphed as the cumulative percent of adopters which typically follows a growth or S-shaped curve (Fig. 1). Because, diffusion frequently occurs through personal networks and personal 


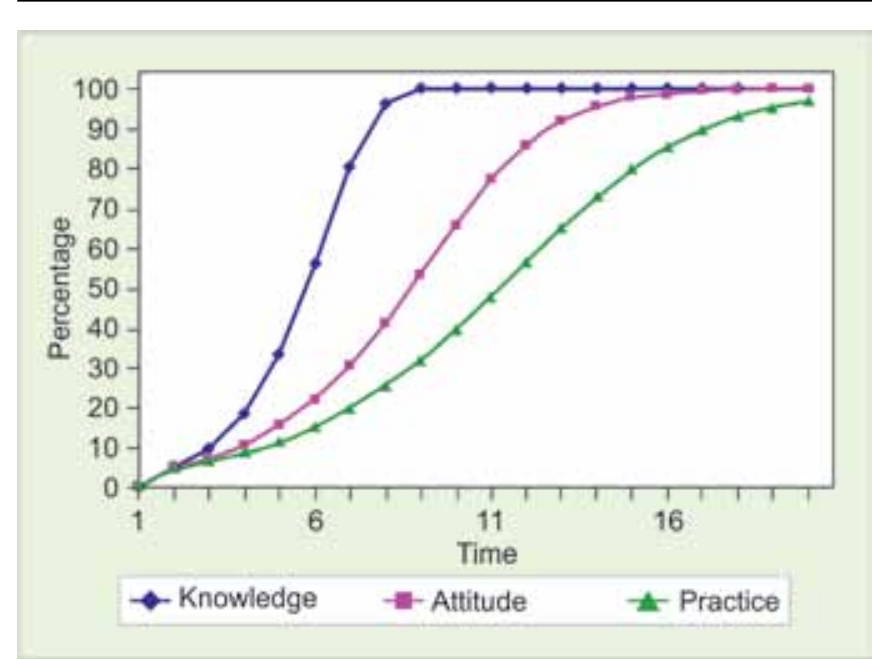

Fig. 1: Typical KAP diffusion curves used to predict the rate of diffusion and the time lag between awareness and use

networks are shaped by ethnic, socioeconomic (SES) factors, and geography, the diffusion of the innovation has a propensity to be shaped by these factors as well. As a result, there may be different diffusion trajectories for different subgroups (e.g. fast diffusion for high SES segments of the population and slow diffusion for low ones). When the diffusion of a new behavior occurs, there are few adopters and the growth in new adopters is slow. Research has found that individuals who adopt an innovation early ('early adopters') are more often persuaded by mass media and other targeted media that provide information that is relevant to the behavior. It is also believed that these early adopters are occasionally free from social norms that would otherwise inhibit them from adopting a new behavior. Whatever the case may be, because new behaviors are uncertain and risky, early adopters have to perceive some reason or benefit in order to adopt the new behavior.

The stages of adoption and the time needed for the diffusion of the new behavior to occur are graphed in Figure 1. The figure illustrates projected rates of the spread of awareness (knowledge), positive attitude (attitude), and behavior (practice). Expected levels for each can be determined by looking at any point in time. The expected lag between knowledge and practice can be attained by reading across the graph (a straightline from any point on the $y$-axis). This general model, where awareness (K) eventually leads to a positive attitude (A), which in turn leads to use of the behavior $(\mathrm{P})$, is known as the learning hierarchy. ${ }^{9}$ Even though this K-A-P sequence occurs often, other researchers argue that different sequences are possible. ${ }^{10}$ For example, some behaviors may be adopted before knowledge of or positive attitudes toward that behavior are developed. For instance, people may use condoms, because they want to protect themselves from pregnancy or sexually transmitted diseases (practice), even though they may not like to use them (low positive attitude), and with little knowledge about their effectiveness (knowledge), thus resulting in the behavioral change sequence to P-A-K. Health promotion and communication campaigns have tried to accelerate behavior change by changing knowledge, attitudes, and directly encouraging people to adopt healthy behaviors, irrespective of the behavior change sequence. Media campaigns serve two specific functions: (1) to spread knowledge rapidly throughout a population so that the knowledge curve grows quickly, and (2) to shorten the K-A-P gap, the time between awareness and use. ${ }^{11}$

\section{NEED OF A KAP SURVEY}

The goal of quantitative methods of data collection is to quantify and measure a phenomenon through the use of questionnaires and statistical processing of the information collected. Questionnaire surveys are the primary method of collecting quantitative data. It includes interviewing, using a questionnaire, a sample of individuals as representative as possible of the entire study population. One advantage of a KAP survey is to allow, in a single survey, the collecting of a large amount of data that will be subject to statistical analysis (which qualitative methods of data collection do not allow).

A KAP survey is a quantitative type method (predefined questions and formatted in standardised questionnaires) that provides access to quantitative and qualitative information. KAP questions tend to reveal not only characteristic traits in knowledge, attitude and behaviors about health related to religious, social, traditional factors, but also the idea that each person has of the body or of disease. These factors are often the source of misconceptions or misunderstandings that may represent obstacles to the activities that we would like to implement and potential barriers to behavior change.

The obstacle to change may be a lack of knowledge of the benefits of health, or lack of knowledge of the problem and its severity. It can also reveal sociocultural or religious representations strongly linked to the change in question or a lack of expertise. Finally, the obstacle to change, resistance or refusal may also be an expression of cultural resistance and/or may reveal a political stance.

Focusing on knowledge and attitudes of the respondents, these questions are intended to identify key knowledge, social skills, and know-how commonly shared by a population or target group about particular issues on which one intends to start a program and/or activities on health education.

A KAP survey can be useful in measuring the extent of a known situation, to confirm or disprove a hypothesis, provide new tangents of a situation's reality; enhancing the knowledge, attitude and practices around specific themes, to identify what is known and done about various subjects 
relating to health; establishing the baseline (reference value) for use in future assessments and will help measure the effectiveness of the activities of health education in changing health behaviors; suggesting an intervention strategy in light of specific local circumstances and the cultural factors that influence them and to plan activities better suited to the respective population involved.

A KAP survey, because it contains very little (or no) open questions, does not, or hardly reveal new problems and deepen the understanding of a situation. A KAP survey essentially records an 'opinion', and is based on the 'declarative' (i.e. statements). In other words, the KAP survey reveals what was said, but there may be considerable gaps between what is said and what is done. ${ }^{12}$

\section{PURPOSE OF KAP SURVEY}

The KAP survey can identify a lack of knowledge, operating procedures or cultural beliefs, thereby enhancing understanding and action targeting improvement in oral health. In a way, this survey can highlight factors which influence 'bad' behavior, such as the reasons behind certain attitudes and the reasons and methods behind certain practices relating to oral health. The KAP survey can also pinpoint communication networks (when or how is information received/disseminated and by whom). These networks are vital for the preparation and dissemination of prevention messages. KAP surveys are used to identify needs and problems. Obstacles can also provide solutions to improve the quality and accessibility of oral health care projects. The KAP survey and, more generally, the dissemination of its findings, provides an occasion to pool different organization to take active part in improving the oral health of the community (local and international NGOs, government, MACs, associations and communities).

The collected data enable these organizations to:

1. Create a database on oral health knowledge levels and measure resulting changes.

2. Set oral health priorities (to work on the most prevalent problem or to identify specific subgroups whose prevention needs differ from those of other groups).

3. Estimate the resources required for the different activities.

4. Select the most effective communication networks and messages.

5. Highlight the scope of the problem, and thereby raise awareness about the need for resources.

\section{THE FREQUENCY OF KAP SURVEYS}

The more frequently a KAP survey is implemented, they more effective it can be in oral health sector. Firstly, by measuring changes in knowledge, attitudes and practices every $2 / 3$ years at most, it provides an element of objectivity for health education teams about their practices and enables them to shift the focus of their action to suit the real needs of populations affected. Secondly, when several quantitative KAP surveys have been previously conducted, the KAP survey can take a more qualitative form and inform individuals involved in health education about what renders prevention messages ineffective. In other words, why certain populations have very good knowledge of oral health but continue to adopt dangerous behavior. A frequently conducted KAP survey provides an up-to-date database about knowledge, attitudes and practices, as well as about more qualitative information on the problems facing populations affected by dental diseases.

AKAP survey requires not only internal human resources, but also the specific skills of an external specialist. Technical advisors or project managers themselves are often too busy to take charge of certain aspects of the KAP survey and the support of a methodological assistant at the outset then at certain key points of the process can be a great help to them. It may be necessary to collaborate with individuals or agencies to determine the sampling (the number of people and zones to be interviewed), create/adapt questionnaires, conduct interviews in the local language and enter or analyse data. ${ }^{12-14}$

\section{TYPES OF KAP SURVEYS}

- To explore an issue: (Baseline KAP) the goal of an exploratory survey is to gather information on the particular population or on a little known subject. This type of survey tends to lean more toward qualitative data than quantitative data to provide statistics. The preparation of questions is one of the major keys.

- To test a hypothesis: (Evaluative KAP) the KAP survey can be used to test the acceptability of messages or a proposed intervention strategy. It is important to ensure that the questions correspond to activities which are possible to put in place.

- To build a database: (Comparative KAP) data are collected at a ' $\mathrm{T}$ ' moment. Data can then be collected again at a moment $\mathrm{T}+1$ to compare, measure or evaluate change. Sampling must be rigorously designed so that $\mathrm{T}$ data can be compared to $\mathrm{T}+1$ data. To compare one's own work to someone else's, similar definitions must be used and it must be clearly specified how this population/survey is similar/different from other populations/ surveys which have been assessed.

To compare two studies on a single population, the conditions for data collection must be strictly comparable (population, sampling, environment, risk factors, health indicators, etc.). Only the risk or protection factor studied 
can be different, which is precisely the challenge of the comparison. There is no ideal situation, outside of so-called experimental surveys. We can therefore accept differences between the two surveys, subject to sufficient reasoning.

We can often have different objectives in mind, such as collecting information for the formulation and dissemination of prevention messages and at the same time creating a database on the level of knowledge and behavior regarding oral health. This can be beneficial but having two different objectives can also lengthen our survey and make it more complex. When the survey has several objectives (baseline and evaluative, for example), it is advised to set priorities and to avoid handling too many aspects in a single survey as it may damage results linked to the general objectives. Surveys with too many research purposes, 'target populations' or with sweeping considerations are unfeasible and quickly become impossible to transpose into a single survey. The simpler the purpose, the more chance the results have of being clear and viable. It is strongly advised to write one or two sentences on the objectives of the survey. This written document can be consulted during data analysis, and more importantly can be used as a basis for discussions and reviews with the different players involved in the KAP survey (technical advisors, stakeholders, consultants, KAP team). ${ }^{9,13-15}$

\section{KAP RESEARCH PROTOCOLS}

A research protocol is a description that describes a methodology, i.e. how a research study is designed, administered and analyzed. The basic elements of a KAP study include:

- Domain identification: The domain is the subject for which the KAP study will be conducted, for example, water quality impairment (e.g., chlorides or phosphorus in a local stream). More specifically, the domain would be the knowledge, attitudes and practices of a community in regard to that impairment.

- Identification of the target audience: The first step in conducting a KAP survey is the selection of the sample to which the survey will be given. The division of the population into smaller categories is typically desirable, as different groups in a community have varying educational, cultural and socioeconomic backgrounds and therefore will likely have differing levels of KAP. The audience targeted by a water quality agency would be the sample population for the KAP survey. In Minnesota, the majority of water quality projects have target audiences under 200 people; although there is a wide range of audience size.

- Determination of sampling methods: A standard method for conducting the survey should be decided upon in advance, and should be consistent for each category to ensure that differences in the result are independent of the sampling method and depend solely on the characteristics of the population in question. KAP sampling methods typically use a questionnaire through interviews (in-person, phone, or mail).

- Analysis and reporting: KAP surveys are very much like a standard social survey, although they are much more focused and limited in scope. KAPs are also uniquely tailored to a specific location, project, or problem. Therefore, they collect the least amount of information to determine whether KAPs have changed from one time period into another. After collection, the data are analyzed to determine the KAP level of the target audience. In larger populations SAS, SPSS, Excel or other standard databases can be used to analyze and present KAP data. For smaller samples, Survey Monkey or a similar Internet-based program is ideal. Knowledge questions, which often have more than one component to a 'correct' answer, must be analyzed differently from those in the attitudes section, which must in turn be analyzed differently from those in the practices section. Reporting of findings is typically done in the presentation of descriptive statistics in table format for each section (knowledge, attitudes, practices). A KAP report should include an introduction, study design, findings, analysis and conclusions. Format and distribution depends upon the terms of reference of the study as defined by the commissioning agency. Most KAP reports contain detailed frequency data and histograms with attributes of the surveyed population. Most also clearly track pre/post intervention knowledge, attitudes and practices over time. ${ }^{3}$

\section{APPROPRIATENESS AND CHALLENGES OF KNOWLEDGE, ATTITUDE AND PRACTICE SURVEYS}

KAP surveys are focused evaluations that measure changes in human knowledge, attitudes and practices in response to a specific intervention. The KAP survey was first used in the fields of family planning and population studies in the 1950s. KAP surveys use fewer resources and tend to be more cost-effective than other social research methods, because they are highly focused and limited in scope. KAP studies tell us what people know about certain things, how they feel, and how they behave. Each study is designed for a specific setting and issue. 'The attractiveness of KAP surveys is attributable to characteristics, such as an easy design, quantifiable data... concise presentation of results, generalisability of small sample results to a 
wider population, cross-cultural comparability, speed of implementation, and the ease with which one can train numerators. ${ }^{, 16}$ In addition, KAP surveys bring to light the social, cultural and economic factors that may influence health and the implementation of public health initiatives. 'There is increasing recognition within the international aid community that improving the health of poor people across the world depends upon adequate understanding of the sociocultural and economic aspects of the context in which public health programs are implemented. Such information has typically been gathered through various types of cross-sectional surveys, the most popular and widely used being the KAP survey., ${ }^{17}$

\section{KAP SURVEYS AND DENTAL PUBLIC HEALTH}

KAP survey data are essential for informing public health work. For example, with regard to dental diseases, a KAP survey can gather information about what respondents know about dental health and disease, what they think about the diseases, the health system response to various dental diseases, and what care someone with dental diseases should seek. KAP surveys are very helpful for identifying knowledge gaps, cultural beliefs, or behavioral patterns that may facilitate or create barriers to dental health or other public health efforts. In addition, the data collected from KAP surveys enable program managers to set dental health priorities, to establish baseline levels, and to measure change from interventions. A KAP survey can be conducted at any point during a public health intervention, but this type of survey is most useful when conducted in the early phases of the project and again after the intervention is completed.

KAP surveys have also been used to assess and improve the condition of reproductive health in developing countries. Additionally, KAP surveys are relevant to public health awareness campaigns. 'Before beginning the process of creating awareness in any given community, it is first necessary to assess the environment in which awareness creation will take place... Understanding the levels of KAP will allow for a more efficient process of awareness creation as it will allow the program to be tailored more appropriately to the needs of the community.' KAP surveys are also 'useful tools for identifying the technological interventions which are important in an area and which are likely to create a significant impact. By analyzing the words farmers use to communicate their KAP in regard to specific elements of a farming system, it is possible to identify those elements which may be good, those which may need to be improved, or those which may need to be discouraged.' With this information, interventions can be more effectively designed. ${ }^{17}$

\section{THE SHORTCOMINGS OF KAP SURVEYS}

\section{Data can be Hard to Interpret Accurately}

One of the main shortcomings of KAP surveys is that it is difficult to ensure an accurate interpretation of data. Researchers should be very cautious regarding the interpretation of results. The reliability of the data can be frequently impacted by underlying contextual and cultural factors. This illustrates the importance of being aware of the respondents' cultural backgrounds when interpreting their responses. ${ }^{18}$

\section{Lack of Standardized Approach to Validate Findings}

Most KAP surveys utilize household surveys. It is also important to consider the fact that social norms and pressures may bias reporting and that conducting household surveys may systematically exclude portions of the population. It is necessary for all KAP surveys to include a validity analysis, so as to ensure the accuracy of the surveys and allow for comparison of the quality of different KAP surveys. ${ }^{19}$

\section{Analyst Biases in KAP Surveys}

KAP surveys have not undergone extensive methodological scrutiny relative to the number of surveys conducted and their importance for social policy. Though, KAP surveys are administered in many countries, the results are almost exclusively analyzed by Western researchers. 'This fact suggests that technical proficiency and therefore, the quality of data generated clearly are considered more important to the process of analysis than familiarity with culture of data origin. That given survey data may derive from cultures and languages different from that of the analyst's own has been ignored as a potential methodological problem. ${ }^{, 20}$ This is problematic since it is likely that the interpretations of KAP survey research data vary depending on the analyst's degree of familiarity with the cultures and practices in the place of data origin. A study conducted in Bangladesh focused on this issue and analyzed differences in responses and interpretations to KAP surveys depending on the analyst's exposure to Bangladeshi culture. The researchers found that 'the Bengali analyzes tended to be more directly relevant to program and policy development. The Bengali groups, in contrast to the Western groups, gave interpretations of the observed empirical relationships that dug beneath the superficial and external features of the problem (e.g. rates and facilities) to lay bare the basic causes of the problem.' Thus, this study illustrates the importance of having analysts who are familiar with the culture and language of the country where the KAP surveys take place. 'The findings of this study demonstrate that indigenous analysts tend to provide analyzes that not only encompass the more context-free analyzes provided 
by foreign analysts but also contain information more directly related to the culture, which provide a flavor of the context. ${ }^{17}$

\section{OTHER CRITICISMS}

A main criticism of KAP survey is that their findings generally lead to prescriptions for mass behavior modification instead of targeting interventions toward individuals. ${ }^{21}$ Other major problems with KAP surveys are that investigators use the surveys to explain health behavior under the assumption that there is a direct relationship between knowledge and action. ${ }^{22}$ In addition, though KAP surveys provide descriptive data about practices and knowledge, they fail to explain why and when certain treatment practices are chosen. In other words, they fail to explain the logic behind treatmentseeking practices. ${ }^{23}$

\section{ALTERNATIVE}

KAP surveys can be useful when the research plan is to obtain general information about public health knowledge and sociological variables. However, 'if the objective is to study health-seeking KAP in context, there are suitable ethnographic methods available, including focus group discussions, in-depth interviews, participant observation, and various participatory methods.' The preferred use of qualitative surveys and research is corroborated by a study on malaria control in Vietnam which found that though the KAP survey generated useful findings, an initial, qualitative investigation (e.g. observation and focus group discussions) to explore the large numbers of potential influences on behavior and exposure risk would have provided a more robust underpinning for the design of survey questions. This would have strengthened its validity and generated additional information. A study conducted by Agyepong and Manderson (1999) also confirms this notion and argues 'that truly qualitative methods, such as observation, individual semistructured interviews, or focus group discussions, are vital foundations for exploratory investigations at the community level, and should precede and underpin populationlevel approaches, such as KAP surveys. ${ }^{24}$

\section{CONCLUSION}

The survey is critical to designing public health interventions and assessing their impact. There are a variety of different methodologies that can be used when designing surveys: open-ended questions, partially categorized questions, and closed ended questions. Each type of question has its own benefits and drawbacks, though partially categorized questions are considered to yield the most accurate and reliable data. KAP surveys explore respondents' KAP toward a particular topic. They are typically used for documenting community characteristics, KAP that may serve to explain health risks and behaviors. Though, they are very useful for obtaining general information about sociological and cultural variables, they are of limited validity if not grounded upon an initial qualitative research study or survey. ${ }^{17}$

\section{REFERENCES}

1. Handicap International, Knowledge, attitudes and practices in risk education: carrying out KAP surveys, 2009, available at: http://www.handicap-international.org.

2. Kaliyaperumal K. Guideline for conducting a knowledge, attitude and practice (KAP) study. Community Opthalmology 2004;4(1):33-37.

3. Knowledge, attitude and practices (KAP) studies for water resources projects. Accessed on $11 \mathrm{Sep}, 2011 .<$ http://files.dnr. state.mn.us/assistance/grants/community/6kap_summary.pdf>

4. Badran IG. Knowledge, attitude and practice the three pillars of excellence and wisdom: a place in the medical profession. Eastern Mediterranean Health J 1995;1(1):8-16.

5. Rogers EM. Diffusion of Innovations. 5th ed. New York: Free Press 2003.

6. Rogers EM, Storey JD. Communication campaigns. In: Berger $\mathrm{CR}$, Chaffee SH, editors. Handbook of Communication Science. Newbury Park, Ca: Sage 1987.

7. Prochaska JO, DiClemente CC. Toward a comprehensive model of change, in: Miller WR Neather N, editors. Treating Addictive Behaviors: Processes of change. New York: Plenum 1986.

8. Valente TW. Evaluating health promotion programs. Oxford: Oxford University Press 2002.

9. Valente, Thomas W, Myers, Raquel. The messenger is the medium: Communication and diffusion principles in the process of behavior change, estudios sobre las culturas contemporaneas, 2010;16(31):249-276.

10. Chaffee SH, Roser C. Involvement and the consistency of knowledge, attitudes and behaviors. In: Communication Research 1986;13(3):373-399.

11. Valente TW, Paredes P, Poppe PR. Matching the message to the process: the relative ordering of knowledge, attitudes, and practices in behavior change research. In: Human Communication Research 1998;24:366-385.

12. MdM, Guide Data collection $>>$ Quantitative methods. The KAP model (Knowledge, attitude and practice), 2009, available on the MdM internet.

13. Mehta M. Behaviour Sciences in Medical Practice. 1st ed. 1998, Jaypee Publisher.

14. WHO, Advocacy, communication and social control for TB control. A guide to developing knowledge, attitude and practice surveys, 2008, available at: http:/www.stoptb.org/assets/documents/resources/publications/acsm/ACSM_KAP\%20GUIDE. pdf.

15. Handicap International, knowledge, attitudes and practices in risk education: carrying out KAP surveys, 2009, available online at: http://www.handicap-international.org. Uk/Resources/Handicap\%20International/PDF\%20Documents/HI\%20Associations/ KAPRiskEducation_2009.pdf.

16. Launiala A. How much can a KAP survey tell us about people's knowledge, attitudes and practices? Some observations from medical anthropology research on malaria in pregnancy in Malawi. Anthropology Matters. 11.1 (2009). Accessed on 
14 Dec 2010.<http://www.anthropologymatters.com/index. php?journal $=$ anth_mattersandpage $=$ articleandop $=$ viewArticle andpath[] $=31$ andpath []$=53>$

17. Survey methodologies. Accessed on $17 \mathrm{Sep}, 2011 .<\mathrm{http}: / / \mathrm{www}$. unitefor sigh.org/global health-university/survey-methodologies.

18. Launiala A. How much can a KAP survey tell us about people's knowledge, attitudes and practices? Some observations from medical anthropology research on malaria in pregnancy in Malawi. Anthropology Matters. 11.1 (2009). Accessed on 14 December2010. $<$ http://www.anthropologymatters.com/index. php?journal $=$ anth_mattersandpage $=$ articleandop $=$ viewArticle andpath[] $=31$ andpath []$=53>$

19. Schopper D, Doussantousse S, Orav J. Sexual behaviors relevant to HIV transmission in a rural African population. How much can a KAP survey tell us? Soc Sci Med 37.3 1993:401-412. Accessedon 14 December 2010.

20. Ratcliffe J. Analyst Biases in KAP Surveys: A cross-cultural comparison. Studies in family planning. Accessed on $13 \mathrm{Dec}$ 2010. $<$ http://www.jstor.org/stable/1965827>
21. Smith H. On the limited utility of KAP-style survey data in the practical epidemiology of AIDS, with reference to the AIDS epidemic in Chile. Health Transit Rev. 3.1 1993;1-16. Accessed on 13 December 2010. <http://www.ncbi.nlm.nih.gov/ pubmed/10148795>

22. Launiala A. How much can a KAP survey tell us about people's knowledge, attitudes and practices? Some observations from medical anthropology research on malaria in pregnancy in Malawi. Anthropology Matters. 11.1 (2009). Accessed on 14 Dec 2010. <http://www.anthropologymatters.com

23. Launiala A. How much can a KAP survey tell us about people's knowledge, attitudes and practices? Some observations from medical anthropology research on malaria in pregnancy in Malawi. Anthropology Matters. 11.1 (2009). Accessed on 14 Dec 2010. $<$ http://www.anthropologymatters.com

24. Quy Anh N. 'KAP surveys and malaria control in vietnam: findings and cautions about community research. Southeast Asian J Trop Med Public Health 36.3 2005:572-577. Accessed on 13 December 2010. 